Determining the Equation of State Isentrope in an Isochoric Heated Plasma

M.E. Foord, D.B. Reisman, P.T. Springer

October 23, 2003

Review of Scientific Instruments 
This document was prepared as an account of work sponsored by an agency of the United States Government. Neither the United States Government nor the University of California nor any of their employees, makes any warranty, express or implied, or assumes any legal liability or responsibility for the accuracy, completeness, or usefulness of any information, apparatus, product, or process disclosed, or represents that its use would not infringe privately owned rights. Reference herein to any specific commercial product, process, or service by trade name, trademark, manufacturer, or otherwise, does not necessarily constitute or imply its endorsement, recommendation, or favoring by the United States Government or the University of California. The views and opinions of authors expressed herein do not necessarily state or reflect those of the United States Government or the University of California, and shall not be used for advertising or product endorsement purposes. 


\title{
Determining the equation-of-state isentrope in an isochoric heated plasma
}

\author{
M.E. Foord, D.B. Reisman, P.T. Springer \\ University of California, Lawrence Livermore National Laboratory, \\ Livermore, CA 94550
}

\begin{abstract}
$\underline{\text { Abstract }}$
A novel method for determining the equation-of-state (EOS) along the release isentrope in an isochoric (constant volume) heated plasma is presented. The sensitivity of this approach is demonstrated using two different equation-of-state models for a solid density, $10 \mathrm{eV}$ expanding Al plasma. Determining the material EOS data is validated to pressures near 80 Mbar, much higher than current isentropic compression experiments allow. Limitations at high temperature $\left(T_{e} \geq 100 \mathrm{eV}\right)$ due to the formation of a radiative conduction layer near the rarefaction interface are also illustrated.
\end{abstract}


The equation-of-state (EOS) of hot dense plasma is of fundamental importance in many areas of research such as in astrophysics, inertial confinement fusion, and strongly coupled plasmas. The plasma motion and macroscopic properties generally depend on the EOS through thermodynamic relations. In the case considered here of a strongly coupled dense plasma, the EOS is highly non-ideal and is typically unknown or may be ill-defined. In this work it is shown that without using the EOS one can obtain pressure-density isentrope curves, $p(\rho)$ experimentally. The concept is to arrange for a uniform deposition of energy into a uniform medium like a thin foil, creating a solid density, uniform plasma. As the plasma undergoes self-similar isentropic expansion (i.e. uncomplicated by heat transport or radiation transport), any of the time-resolved density profiles at subsequent times can be analyzed to give the particular pressure-density isentrope corresponding to the initial conditions.

One method for obtaining material EOS data is through isentropic compression experiments (ICE). In this approach, a rising pressure pulse is applied to a solid density sample. The pressure isentrope is then inferred from velocity measurements at the back surface as the compression wave passes through the sample. ${ }^{1,2}$ However, this technique has been generally limited to pressures near a few Mbars. ${ }^{3}$ In this Brief Communication, the method we develop is validated to much higher pressures $(80 \mathrm{Mbar})$ and temperatures $(100 \mathrm{eV})$. The sensitivity of the technique is illustrated using two equation-of-state models in hydrodynamic simulations where a thin Al foil is isochorically (constant volume) heated. While the equations and general solution for isentropic expansion are well know ${ }^{4}$, the derivations presented here extend the previous work by deriving the pressure-density $P(\rho)$ relationship for an arbitrary EOS from a single measurement of the density profile.

A promising new approach for isochoric heating involves the interaction of a sub-ps petawatt laser with a thin foil, which routinely generates a high flux of protons with energies $E=5-20 \mathrm{MeV} .{ }^{5,6}$ Due to their Coulomb interaction with free and bound electrons and their long 
range, these $\mathrm{MeV}$ protons can rapidly ionize and heat relatively thick samples to hundreds of Mbar pressure. Experiments have already demonstrated that protons produced in this manner can heat $10 \mu \mathrm{m} \mathrm{Al}$ foils to temperatures $\mathrm{T}>20 \mathrm{eV} .{ }^{7}$ Shown in Fig. 1 are simulated density and temperature profiles of a $20 \mu \mathrm{m}$ thick Al foil heated with a $10 \mathrm{MeV}$ proton beam. The temperature is seen to vary less than $1 \%$ over the bulk of the foil.

Consider a uniformly heated thin foil of density $\rho(x)=\rho_{0},|x|<x_{o}$; $\rho=0,|x|>x_{o}$. For clarity, we begin with the well known equations of continuity and motion ${ }^{4}$ :

$$
\begin{gathered}
\frac{\partial \rho}{\partial t}+\frac{\partial}{\partial x}(\rho u)=0 \\
\frac{\partial u}{\partial t}+u \frac{\partial u}{\partial x}=-\frac{1}{\rho} \frac{\partial p}{\partial x}
\end{gathered}
$$

where $p, \rho$, and $u$ are the total pressure, mass density and velocity of the plasma, respectively.

For isentropic flow: $\partial p /\left.\partial x\right|_{S}=\partial p /\left.\partial \rho\right|_{S} d \rho /\left.d x\right|_{S} \equiv C_{S}^{2} \partial \rho / \partial x$.

This is an important step in allowing the sound speed to be related to local pressure gradient, and is only true in general for the case of isentropic flow.

The equation of motion is then rewritten as:

$$
\frac{\partial u}{\partial t}+u \frac{\partial u}{\partial x}=-C_{S} \frac{\partial \rho}{\partial x} .
$$

Eq. 3 is based on the assumption that each fluid element begins with the same entropy and expands adiabatically, ie., thermal energy is 
only lost due to $\mathrm{pdV}$ work. The validity of this assumption is further discussed below.

Assuming self-similar flow, (valid for $t<x_{o} / C_{S}$ ) we define $\rho=f(\xi)$, and $u=g(\xi)$, where $\xi \equiv x / t$, and define derivatives with respect to $\xi$ as $f^{\prime}$ and $g^{\prime}$.

After substitution, Eqs. 1 and 3 become:

$$
\begin{aligned}
& f^{\prime}(g-x / t)+f g^{\prime}=0 \\
& g^{\prime}(g-x / t)+C_{S}^{2} \frac{f^{\prime}}{f}=0 .
\end{aligned}
$$

Further substitution yields the known solution $^{8}$ for the sound speed $C_{S}(x, t) ; x>0$ :

$$
C_{S}=g-x / t
$$

and a relationship between the density and the sound speed

$$
f^{\prime} C_{S}+f\left(C_{S}^{\prime}+1\right)=0 \quad \text { or } \quad\left(f C_{S}\right)^{\prime}=-f .
$$

Recalling that for some function $h(\xi)$,

$$
\frac{\partial h}{\partial x}=\frac{d h}{d \xi} \frac{\partial \xi}{\partial x}=\frac{d h}{d \xi} \frac{1}{t}=\frac{h^{\prime}}{t},
$$

Eq. 7 is rewritten as;

$$
\frac{\partial}{\partial x}\left(\rho C_{S}\right)=-\frac{\rho}{t}
$$

Eq. 8 relates the sound speed $C_{S}$ to the density $\rho$ at time $t$ and is key to determining the pressure isentrope. The sound speed profile 
can therefore be determined from a single density profile measurement by integrating the density profile:

$$
C_{S}(x, t)=\frac{-1}{\rho t} \int_{x_{R}}^{x} \rho d x+\frac{\rho_{o} C_{S o}}{\rho}
$$

where $x_{R}$ is the position of the rarefaction interface at time $t$, and $\rho_{o}$ and $C_{S o}$ are the density and sound speed in the unperturbed material, respectively. In solving for $C_{S}(x)$ it is important to chose $x_{R}$ at a position near the rarefaction interface where the density begins to sharply drop, in order to avoid residual errors. $C_{S}(x)$ is very sensitive to cancellation from the two terms on the RHS of Eq. 9 in the outer low density region of the profile. If $C_{S o}$ or $t$ are not accurately measured, unrealistic large positive or even negative values of $C_{S}(x)$ could result in the low density region. In order to overcome this difficulty, we set $\rho C_{S}$ to zero at the lowest density point on the profile, defined here as $x_{\max }$.

Eq. 9 is rewritten in its approximate form;

$$
C_{S}(x, t) \cong \frac{-1}{\rho t} \int_{x_{R}}^{x} \rho d x+\frac{1}{\rho t} \int_{x_{R}}^{x_{\max }} \rho d x=\frac{1}{\rho t} \int_{x}^{x_{\max }} \rho d x .
$$

Since $C_{S}\left(x_{\max }\right)$ is actually finite, this approximation introduces a small error near $x_{\max }$. However, as shown below, the region near $x_{\max }$ contributes little to the pressure integral. Thus, Eq. 10 provides a useful and robust approximation for determining the sound speed profile and does not require an independent measurement of $C_{S o}=x_{R} / t$ (see Eq. 6). In fact, $C_{S o}=C_{S}\left(x_{R}\right)$ is now determined directly from Eq. 10.

The pressure can then be determined by integrating the sound speed squared:

$$
p=\int_{x}^{x_{\max }} C_{S}^{2} \mathrm{~d} \rho=\int_{x}^{x_{\max }} C_{S}^{2} \frac{\partial \rho}{\partial x} \mathrm{~d} x
$$


determining the $p(\rho)$ isentrope. This integral is dominated by the high pressure region near the interface where the sound speed is the largest and where $\partial p / \partial \rho$ can be determined most accurately.

This method is applied to simulations of an expanding thin Al foil, assumed to be uniformly heated to $10 \mathrm{eV}$, using the hydrodynamics code LASNEX. ${ }^{9}$ The 1-D simulations consisted of a 250 -zone variablespaced grid which allowed greater resolution near the foil edges, and included electron thermal conduction and radiative transport models appropriate for dense plasma conditions. The EOS model QEOS ${ }^{10}$ was used in the first set of simulations. Shown in Fig. 2 are density and entropy profiles at $\mathrm{t}=400 \mathrm{ps}$. The uniform entropy profile indicates that the expansion is essentially isentropic and that dissipative processes, such as heat conduction and viscosity do not play an important role in the hydrodynamics.

Shown in Fig. 3 are calculated profiles of $C_{S}$ using Eqs. 9 and 10. Also shown is the $C_{S}(x, t=400 \mathrm{ps})$ profile from the simulations. Good agreement is found except near $x=x_{\max }$, where the sound speed is forced to zero by Eq. 10. In Fig. 4, the resulting $p(\rho)$ curves calculated from Eq. 11 are shown for each of the three cases (curves a-c). Good agreement is found, indicating that the contribution to the integral near $x=x_{\max }$ is small. The validity of Eq. 9 and its approximation (Eq. 10) are clearly demonstrated.

Simulations were also performed utilizing a tabular EOS model, constructed of various theoretical calculations and data over a broad density and temperature range. ${ }^{11}$ For comparison, the initial energy density was set equal to the QEOS case. Because of the differences in the specific heats, the tabular model required an initial temperature of $11.0 \mathrm{eV}$ to match the same energy density as QEOS at $10.0 \mathrm{eV}$. The tabular model generated a slighter faster rarefaction wave, as seen in Fig. 2.

A comparisons of the $p(\rho)$ isentropes from the two EOS models is also shown in Fig. 4. The slightly faster rarefaction wave for the tabular model, as seen in Fig. 1 results in a faster rise in $\partial p / \partial \rho$ shown in curve (d). Since the initial temperatures are slightly 
different $(10 \%)$, the curves reach different maximum pressures at solid density. From the geometry, $\Delta \rho / \rho \approx(1 / 2) \Delta C_{S} / C_{S}$, where $\Delta \rho$ is the density difference between models at a point near $x_{R}$. Thus, in order to resolve these two EOS models having sound speeds that differ by $\approx 5 \%$, the density profiles need to be measured with an accuracy $\approx 2.5 \%$.

We mention that at higher temperatures, radiative or electron thermal conduction may be sufficient to affect the isentropic nature of the expansion and produce more isothermal-like profiles, especially near the rarefaction interface. Simulations indicate that for aluminum at $T_{e} \geq 100 \mathrm{eV}$, radiative conduction begins to smooth the density and temperature profiles near the interface. The pressure profile for an expanding $100 \mathrm{eV}, 80 \mathrm{Mbar}$ solid thin aluminum plasma at $2 \mathrm{~ns}$ is shown in Fig. 5. In this case, the density integrals in Eqs. 10 and 11 for $C_{S}$ and $p(\rho)$ respectively, begin outside the conduction region near the inflection point on the density curve, limiting $x_{R}$ to a region a few tens of microns from the peak pressure and sound speed, and thus limiting to some extent the peak pressures that can be determined. As shown in Fig. 5, pressures close to 80 Mbar were obtained, indicating the validity of this approach to almost two orders of magnitude higher pressure than typical isentropic compression experiments allow ${ }^{3}$.

Energetic $(\mathrm{E}>50 \mathrm{keV}) \mathrm{x}$-ray sources are currently being developed for imaging dense structures. ${ }^{12}$ For example, in a partially ionized plasma, bound-free absorption at photon energies above a closed shell mainly depends on the ion density and can thus be used to determine the mass density profiles, independent of the degree of ionization. Previous experiments have demonstrated a few $\mu \mathrm{m}$ and a few ps resolutions in $\mathrm{x}$-ray radiographed shock experiments. ${ }^{13}$ Thus, for the examples given above, where the expansion distances are $15-80 \mu \mathrm{m}$ and the time scales are many hundreds of picoseconds to nanoseconds, the required resolutions may be experimentally feasible. We also mention that there are many materials where essentially no offHugoniot data exists for dense plasma, or where the data varies widely. In these cases, even $10-20 \%$ accurate density and time measurement could provide important new EOS data, currently unavailable by any other means. 
As shown in Fig. 1, protons generated during the sub-ps intense laser pulse can uniformly heat tens of micron thick $\mathrm{Al}$, as required for these experiments. For example, in Fig. 2, $8 \mu \mathrm{m}$ of initially solid density Al has expanded after $400 \mathrm{ps}$, while in Fig. 5, $15 \mu \mathrm{m}$ of the initially solid density Al has expanded after 2 ns. While details of an actual proton spectrum need to be considered as well, these results illustrate the feasibility of producing uniformly heated samples with a sufficiently energetic proton beam.

Depending on the distance to the target (typically a few hundred microns), the typical heating time due to beam spreading is a few ps. Other simulations not shown here indicate that varying the heating time from instantaneous to a few $p s$, has an insignificant affect on the density profile $(<1 \%)$ after a few hundred ps. Thus, the density profile analyzed in Fig. 1 at $t=400$ ps should be insensitive to the ps heating times associated with proton generation.

In summary, a novel method for determining EOS data from density profile measurements in isentropic release experiments is presented. While EOS along the shocked Hugoniot are known to very high pressures, typically little or no off-Hugoniot data exists for the majority of materials in the warm-to-hot dense plasma regime. By varying the initial proton flux, and thus the initial $\left(\rho_{0}, T\right)$ point, release experiments could potentially map out large areas of EOS phase-space. Higher or lower density regimes could likewise be explored with pre-shocked or foam materials, respectively.

\section{ACKNOWLEDGEMENTS}

The authors thank A. Osterheld and G. Collins for helpful discussions. This work was performed under the auspices of the U.S. Department of Energy by the UC/Lawrence Livermore National Laboratory under Contract No. W-7405-Eng-48. 


\section{References}

${ }^{1}$ D. B. Reisman, A. Toor, R. C. Cauble, C. A. Hall, J. R. Asay, M.D. Knudson, M. D. Furnish, J. Appl. Phys. 891625 (2001).

${ }^{2}$ C. A. Hall, J. R. Asay, M. D. Knudson, W. A. Stygar, R. B. Spielman, T. D. Pointon, D. B. Reisman, A. Toor, R. C. Cauble, Rev. Sci. Instr. 72 3587 (2001).

${ }^{3}$ D. B. Reisman (private communication, 2002).

${ }^{4}$ Zel'dovich and Raizer, in Physics of Shock Waves and High-Temperature Hydrodynamic Phenomena, Academic Press, New York, 1967.

${ }^{5}$ R. A. Snavely, M. H. Key, S. P. Hatchett, et al., Phys. Rev. Lett. 852945 (2000).

6 A. J. Mackinnon, M. Borghesi, S. Hatchett, et al., Phys. Rev. Lett. 861769 (2001).

${ }^{7}$ P.K. Patel, A.J. Mackinnon, M.H.Key, T.E. Cowan, M.E. Foord, M. Allen, D.F. Price, H. Ruhl, P.T. Springer, R. Stephens, Isochoric heating of solid-density matter with an ultrafast proton beam, (Accepted PRL, Aug, 2003).

${ }^{8}$ W.L. Kruer, in The physics of laser plasma interactions. AddisonWesley Publishing Co. 1988.

${ }^{9}$ G. B. Zimmerman and W.L. Kruer, Comments Plasma Phys. Controlled Fusion 251 (1975).

${ }^{10}$ R. M. More, K. H. Warren, D. A. Young, and G. B. Zimmerman, Phys. Fluids 313059 (1988).

${ }^{11}$ K. S. Holien, J. Appl. Phys. 59149 (1986).

12 A. J. Mackinnon, Y. Semtoku, P. K. Patel, et al., Phys. Rev. Lett. 88 215006(2002); E. Andersson, G. Holzer, E. Forster, M. Gratz, L. Kiernan, A. Sjogren, S. Svanberg, J. Appl. Phys. 903048 (2001); G. Holzer, E. Forster, M. Gratz, C. Tillman, S. Svanberg, J. X-ray Sci. and Technol. 7, 50 (1997); D. Salzmann, Ch. Reich, I. Uschmann, E. Forster, P. Gibbon, Phys. Rev. E 65036402 (2002). 
13 G.W. Collins, L.B. Da Silva, P. Celliers, D.M. Gold, M.E. Foord, R.J. Wallace, A. Ng, S.V. Weber, K.S. Budil, and R. Cauble, Science 281,1178 (1998). 


\section{Figure Captions}

Fig. 1 Temperature a) and density b) profile simulation of a $20 \mu \mathrm{m}$ Al foil heated by a 1 ps $10 \mathrm{MeV}$ proton beam at $\mathrm{t}=1 \mathrm{ps}$. Proton beam is incident from the right.

Fig. 2 Density profiles at 400 ps for $10 \mathrm{eV}(11 \mathrm{eV})$ solid density plasma using QEOS (tabular) EOS models and the corresponding QEOS specific entropy profile. Expansion is symmetric about $\mathrm{x}=0$.

Fig. 3 Sound speed profiles calculated using Eq. 9 (curve a), Eq. 10 (curve b), and from the hydrodynamic simulation (curve c).

Fig. 4 Calculated $p(\rho)$ isentropes for the three cases shown in Fig. 2 (curves a-c) showing the validity of Eq. 10. Curve $d$ is calculated from the tabular EOS model density profile.

Fig. 5 Expansion profiles $p(\rho)$ and $p(x)$ at $2 \mathrm{~ns}$ for a $100 \mathrm{eV}$ solid density aluminum plasma. Simulated profiles are the solid curves, while the thicker dashed curves are calculated values showing a slight limitation in reaching peak values due to conduction at the rarefaction front, $\mathbf{x}_{\mathbf{R}}$. 


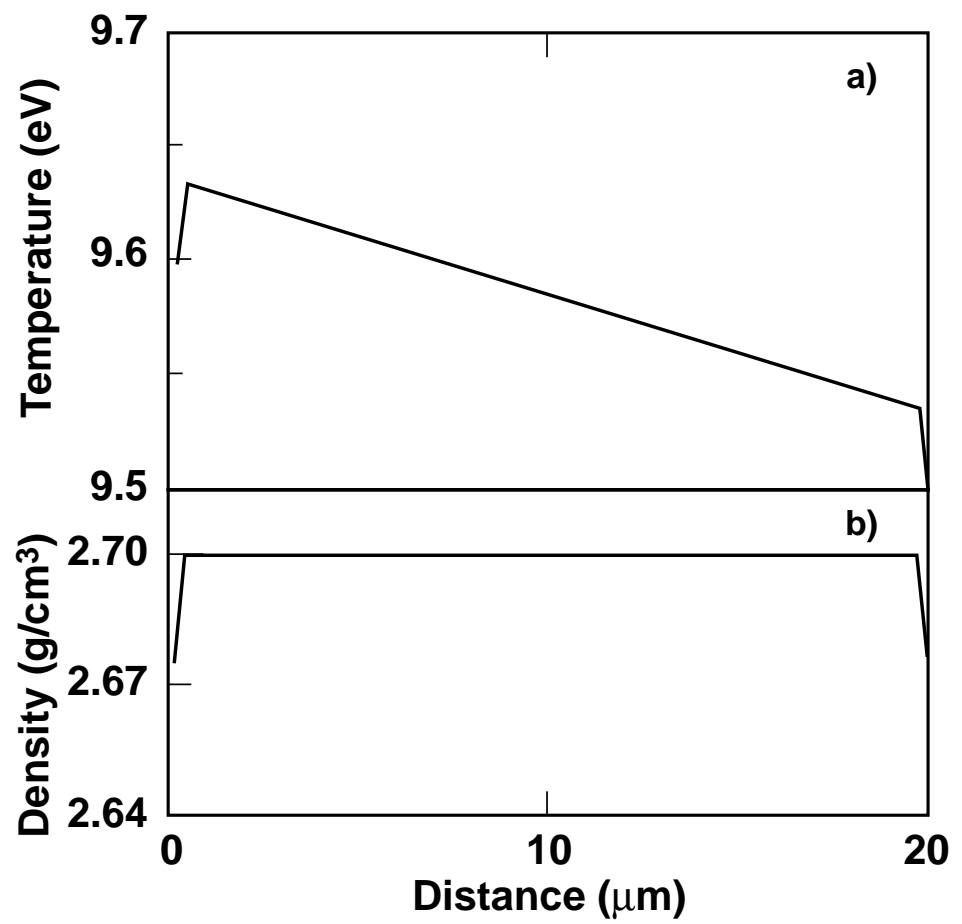

Figure 1 


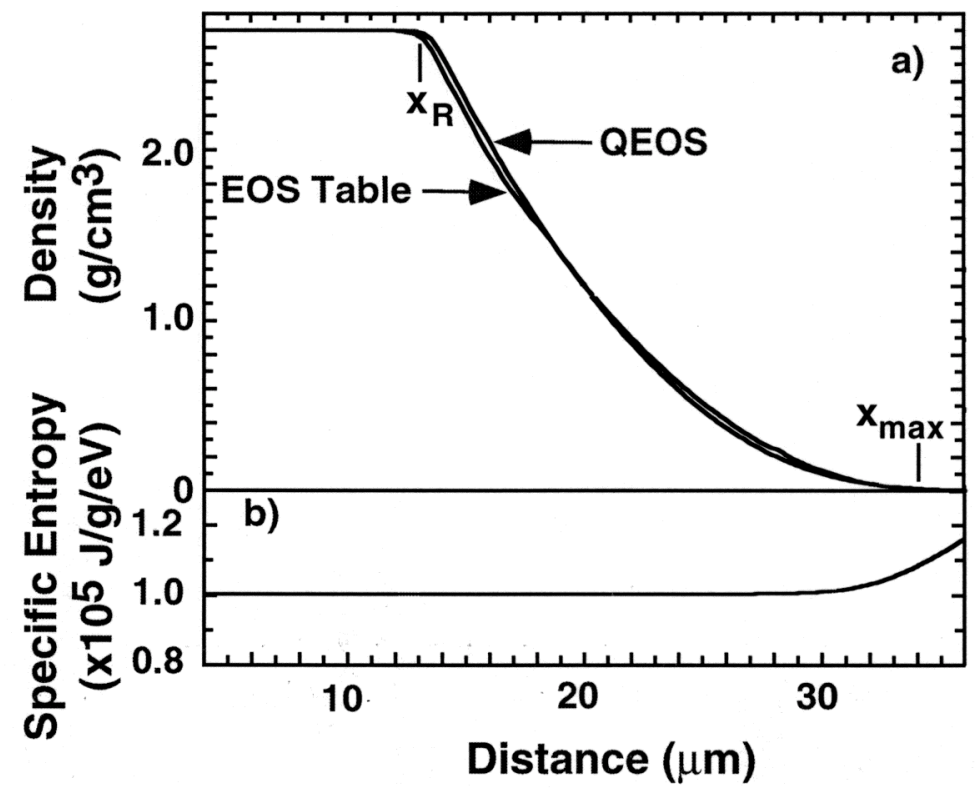

Figure 2 


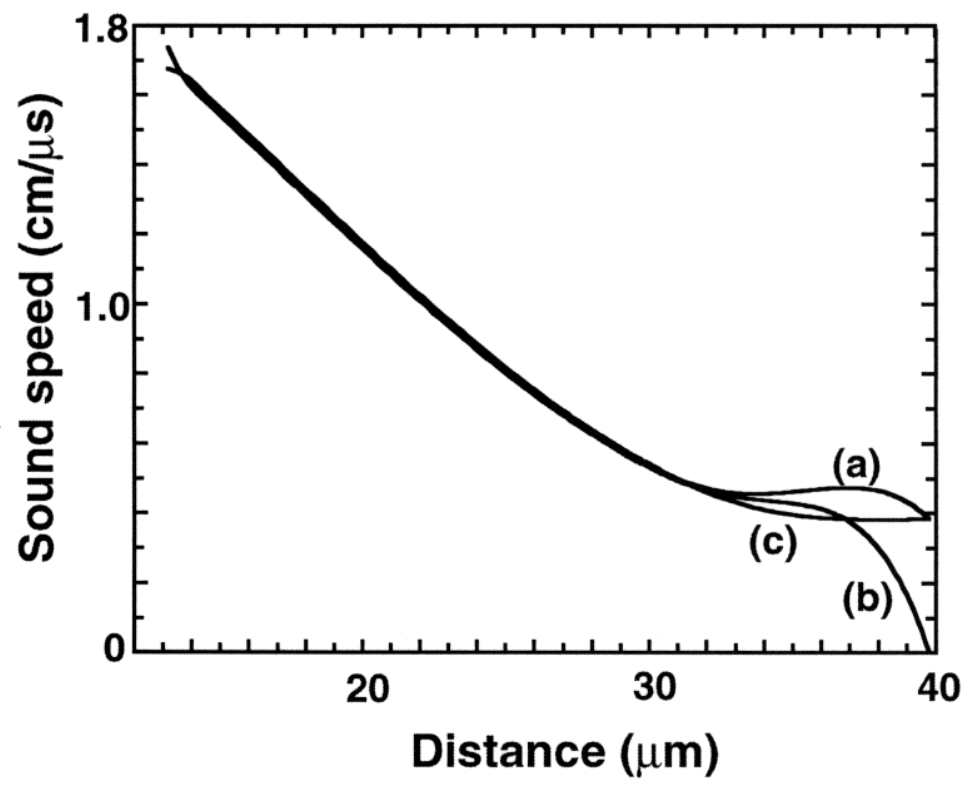

Figure 3 


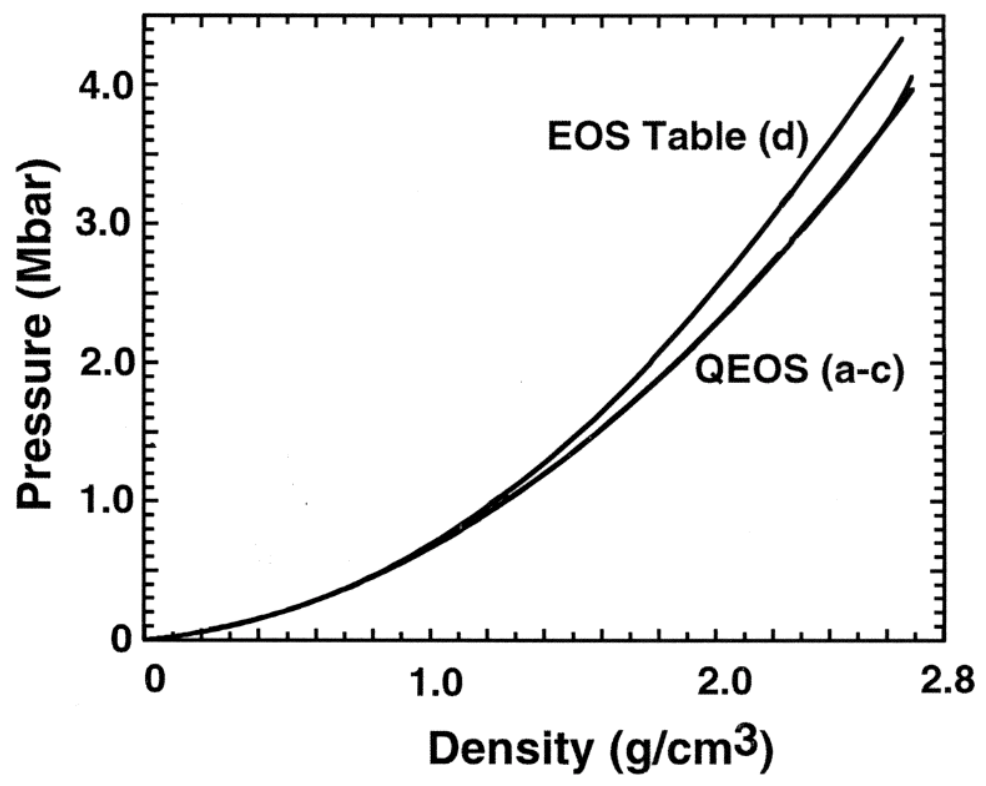

Figure 4 


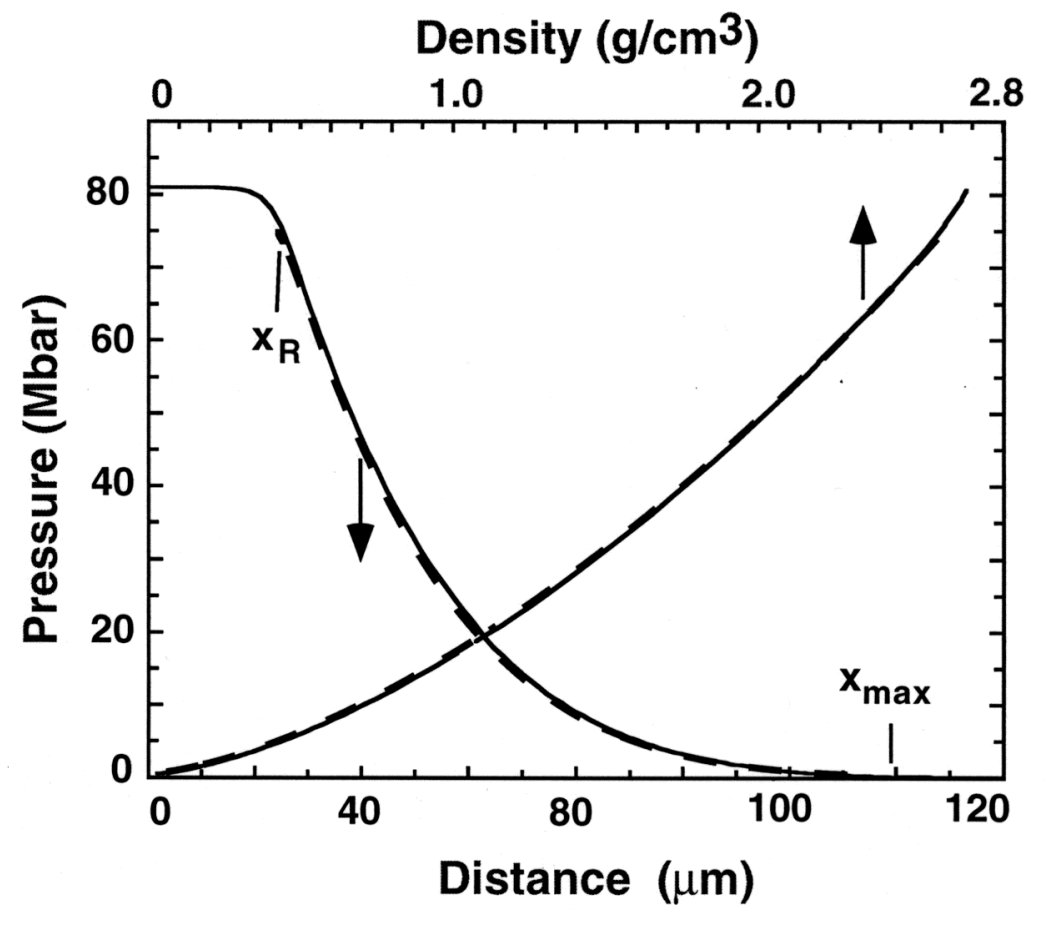

Figure 5 